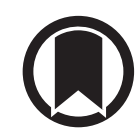

CrossMark

\title{
Primary ciliary dyskinesia patients have the same $P$. aeruginosa clone in sinuses and lungs
}

\author{
To the Editor:
}

Similar to patients with cystic fibrosis (CF) and non-CF bronchiectasis, patients with primary ciliary dyskinesia (PCD) are prone to recurrent or chronic lung infections with Pseudomonas aeruginosa. Chronic $P$. aeruginosa lung infection has a prevalence of up to $39 \%$ in patients with PCD [1] and is associated with structural damage, affecting lung function. Treatment of $P$. aeruginosa infection is challenging because $P$. aeruginosa adapts to the host environment through genotypic/phenotypic changes, promoting a reduced immune response [2]. We have found previously that the paranasal sinuses in patients with $\mathrm{CF}$ act as bacterial reservoirs where $P$. aeruginosa adapts and recolonises $P$. aeruginosa-eradicated lungs $[3,4]$. In addition, our group has reported $P$. aeruginosa-positive cultures from the upper and lower airways of patients with PCD [5]. However, it was unclear whether the paranasal sinuses of patients with PCD also act as bacterial reservoirs. We are investigating whether the same $P$. aeruginosa clone type colonises both the paranasal sinuses and the lungs, and the extent to which $P$. aeruginosa adapts to the host environment via genotypic/phenotypic changes.

From 2009 to 2017, we collected and analysed 38 P. aeruginosa isolates (21 paranasal sinus, 17 lung) from nine chronically lung-infected patients with PCD [6]. At least one sinus isolate and one lung isolate were collected from each patient. The mean time between first and last isolate was 3.0 years (range $0-5.5$ years) (figure 1). Chronic P. aeruginosa infection was diagnosed according to the modified CF Leeds criteria, or when anti- $P$. aeruginosa precipitin levels were elevated in combination with a $P$. aeruginosa-positive sample [1]. Sinus isolates were sampled from the maxillary, ethmoidal, sphenoid or frontal sinuses during endoscopic sinus surgery (ESS). Lung isolates were obtained by bronchoalveolar lavage in combination with ESS or, at a different time, by expectoration or endolaryngeal suction at our PCD centre. Nine of the lung isolates have been described previously by our group [7]. Our principal eradication treatment for $P$. aeruginosa infection in patients with PCD is 3 weeks' inhalation of colistin and oral ciprofloxacin, and the second-line treatment is 2 weeks of intravenous aminoglycoside and $\beta$-lactam. $P$. aeruginosa genotype/ phenotype was obtained from whole genome sequencing, growth rate, motility, protease secretion, biofilm formation and antibiotic susceptibility, and compared to the reference strain PAO1.

Each patient (P1-P9) had one individual P. aeruginosa clone type, which was present in both the paranasal sinuses and the lungs; thus, nine different $P$. aeruginosa clone types were identified (figure 1). P1-P5 and P7 retained their individual clone types for years, with an observed maximum of 5.5 years. P6, P8 and P9 also retained their individual clone types over time, but their sinus isolates were collected simultaneously or within months of the lung isolates. None of the patients shared clone types and only P7's clone type had previously been identified from a patient with CF at our centre. There was no evidence of cross-infection, as DK19 belongs to the environmentally abundant PA14 clonal complex.

All $P$. aeruginosa sinus and lung isolates from each patient's clone type had similar genotypes (figure 1). Isolates from different patients had very different genotypes. P3's clone (DK64) and P7's clone (DK19) had no gene mutations. P1's clone (DK60) was a hypermutator [8] with a total of 37 mutated genes, four of which were shared between sinus and lung isolates. The hypermutator status did not show as

@ERSpublications

For the first time it is shown that the same Pseudomonas aeruginosa clone exists in both the upper and lower airways in patients with PCD, providing a solid support of the unified airway theory where the sinuses are a possible bacterial reservoir http://bit.ly/2kcE9tq

Cite this article as: Arndal E, Johansen HK, Haagensen JAJ, et al. Primary ciliary dyskinesia patients have the same P. aeruginosa clone in sinuses and lungs. Eur Respir J 2020; 55: 1901472 [https://doi.org/10.1183/ 13993003.01472-2019]. 
Patient/P. aeruginosa clone type

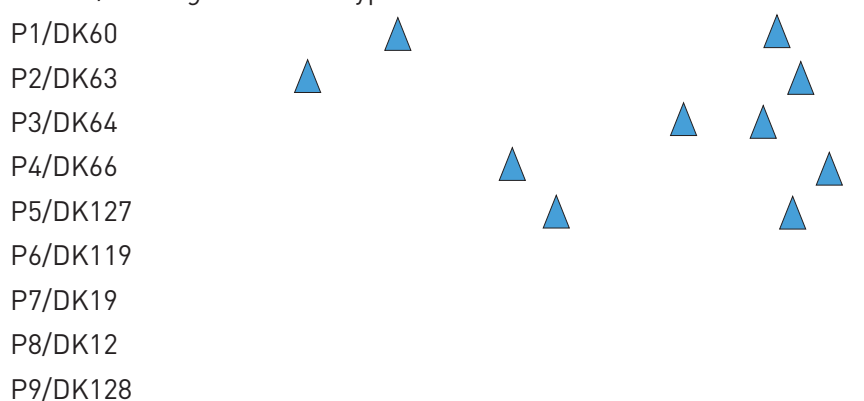

$\triangle$

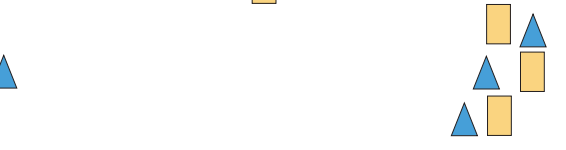

$20092010 \quad 20112013$

b)

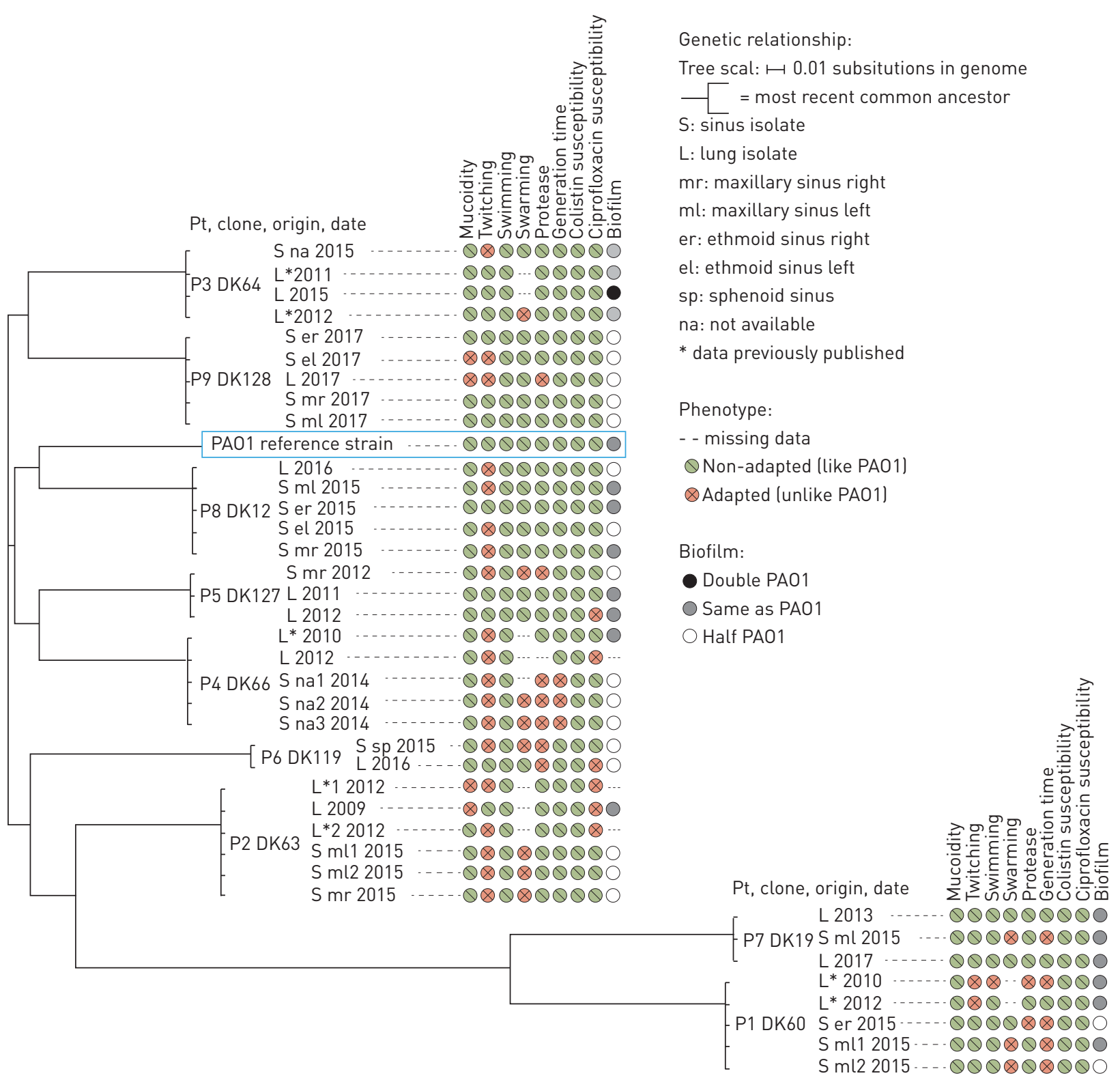

FIGURE 1 Genetic relationship and phenotype of Pseudomonas aeruginosa isolates from the paranasal sinuses and lungs in patients with primary ciliary dyskinesia. a) Date, origin and clone type of $P$. aeruginosa samples in each patient (P1-P9); b) phylogenetic tree showing the genetic relationship between $P$. aeruginosa clones and PAO1 reference strain. Each branch only has isolates from one clone type. Clonal isolates are ordered according to genetic relationship. Circles after each isolate depict the phenotypic characteristics. 
phenotypic changes. The remaining patients' clones had a varying, but low, number of mutated genes (mean 3.5, range 0-10). Some mutations were present in all isolates within a given clone type, while others were only present in some of the isolates. The following mutated genes were found in more than one clone type: MigA (DK66, DK119), involved in biofilm formation and colistin resistance [9]; AlgU (DK63, DK128), affecting production of alginate (biofilm matrix) and associated with a more resilient mucoid phenotype [2]; and LasR (DK60, DK66), with a central regulatory role in quorum sensing, which also improves bacterial survival [2]. We found LasR, AlgU and MigA mutations in some isolates, but saw no consistent phenotypic change in mucoidity or colistin resistance. The phenotypic characteristics were like those of PAO1, with no consistent adaptational patterns between isolates from sinuses and lungs and no clear correlation between genotype and phenotype. This may have been due to other regulatory mechanisms and post-transcriptional events that affect gene expression and gene product. Future analysis of $P$. aeruginosa RNA may clarify this connection.

All the isolates from each patient belonged to the same clone type, with partial yet incomplete genotypic/ phenotypic match, among isolates from the different sinuses and the lungs (figure 1). This limited parallel adaptation may be caused by different local conditions in the sinuses and lungs promoting divergent adaptation of $P$. aeruginosa. Similarly, MARKussen et al. [10] described different evolution of clonal sublineages within the same CF lungs. The genotypic/phenotypic profile in $P$. aeruginosa infection in early $\mathrm{CF}$ is less adapted than in late CF, where different sublineages develop due to temporospatial diversification [10]. We have found that the limited level of adaptation and the PAO1-like phenotype seen in our PCD P. aeruginosa isolates resembles the early $P$. aeruginosa infection in CF $[7,11]$.

Previous literature on P. aeruginosa in CF suggests that intensive antibiotic treatment promotes bacterial adaptation and antibiotic resistance [12]. We found that $100 \%$ of our isolates were sensitive to colistin, despite five isolates having a MigA mutation, and $84 \%$ were sensitive to ciprofloxacin. All sinus isolates were sensitive to ciprofloxacin while six out of 17 lung isolates had intermediate susceptibility. A possible explanation for this high level of antibiotic susceptibility could be that $P$. aeruginosa from patients with PCD experiences less antibiotic stress than in CF. Differences in antibiotic bioavailability and host inflammatory response between the paranasal sinuses and the lungs should also be considered. Dонт et al. [13] reported less effect of i.v. antibiotics on human sinonasal inflammatory markers than in the lungs. KARMA et al. [14] showed that in patients with chronic rhinosinusitis, the antibiotic concentration in sinus fluid was substantially lower than in the sinus mucosa, suggesting an insufficient dose and time inside the paranasal sinus cavities to ensure bacterial death. In CF, oxidative stress promotes bacterial genotypic/ phenotypic adaptation which increases bacterial resistance and thereby a higher likelihood of persistent infection [7]. All the aforementioned stress factors may also influence bacterial adaptation in patients with PCD.

Addressing the unified airways theory, HANSEN et al. [3] showed that patients with CF shared P. aeruginosa clones between the upper and lower airways and Alanin et al. [5] showed the presence of $P$. aeruginosa in cultures from the upper and lower airways and a persistent $P$. aeruginosa clone type in the lungs of patients with PCD. We have previously shown that ESS in combination with postoperative nasal irrigation, nasal steroids and systemic antibiotics can help eradicate $P$. aeruginosa from the paranasal sinuses in patients with CF and PCD $[4,15]$. The aforementioned literature describes $P$. aeruginosa infection initially in the lungs and then later in the sinuses, but is that the true chronological order? Which comes first: lung infection or sinus infection? The hypothesis of a possible sinus focus was generated because of evidence that lung-transplanted CF patients were recolonised with the same pathogen that they had had before lung transplantation. Extracted from RADEMACHER et al. [16], 11 out of 34 lung-transplanted patients with non-CF bronchiectasis continued to be chronically lung-infected with the same pathogen that they had had before lung transplantation, and $P$. aeruginosa was the most common pathogen. Theoretically, the sinus samples in these patients may have been $P$. aeruginosa-positive, but this remains undetermined. However, the exact order of colonisation may be less important than recognising that $P$. aeruginosa colonises both the sinuses and lungs and that eradication treatment should be aimed not only at the lower airways, but at the unified airways. Furthermore, the upcoming European Position Paper on Rhinosinusitis and Nasal Polyps 2020 has an increased interest in multidisciplinary collaborations, focusing especially on the unified airways in PCD and CF.

We have shown for the first time that patients with PCD and P. aeruginosa lung infection harbour the same clone type in their paranasal sinuses and lungs, providing support for the unified airways theory in PCD. As in CF, the paranasal sinus focus in patients with PCD may be responsible for recolonising the lungs, so early eradication of the paranasal sinus colonisation could reduce lung infections. The important role of the upper airways in patients with PCD helps us to better understand the pathogenesis of $P$. aeruginosa infection. We therefore recommend that contributions from otorhinolaryngologists be considered in the development of PCD and CF treatments. 
Elisabeth Arndal $\oplus^{1}$, Helle K. Johansen $\oplus^{2,3}$, Janus A.J. Haagensen ${ }^{4}$, Jennifer A. Bartell $\odot^{4}$, Rasmus L. Marvig $\oplus^{5}$, Mikkel Alanin $^{1}$, Kasper Aanæs ${ }^{1}$, Niels Høiby $\oplus^{2,6}$, Kim G. Nielsen $\oplus^{7}$, Vibeke Backer ${ }^{8}$ and Christian von Buchwald $\oplus^{1}$ ${ }^{1}$ Dept of Otorhinolaryngology - Head and Neck Surgery and Audiology, Copenhagen University Hospital, Rigshospitalet, Copenhagen, Denmark. ${ }^{2}$ Dept of Clinical Microbiology, Copenhagen University Hospital, Rigshospitalet, Copenhagen, Denmark. ${ }^{3}$ Dept of Clinical Medicine, Faculty of Health and Medical Sciences, University of Copenhagen, Copenhagen, Denmark. ${ }^{4}$ Novo Nordisk Foundation Center for Biosustainability, Technical University of Denmark, Lyngby, Denmark. ${ }^{5}$ Center for Genomic Medicine, Copenhagen University Hospital, Rigshospitalet, Copenhagen, Denmark. ${ }^{6}$ Institute of Immunology and Microbiology, Copenhagen University Hospital, Rigshospitalet, Copenhagen, Denmark. ${ }^{7}$ Danish PCD Center, Pediatric Pulmonary Service, Dept of Pediatric and Adolescent Medicine, Copenhagen University Hospital, Rigshospitalet, Copenhagen, Denmark. ${ }^{8}$ Centre for Physical Activity Research (CFAS), Rigshospitalet, Copenhagen University Hospital, Denmark.

Correspondence: Elisabeth Arndal, Dept of Otorhinolaryngology - Head and Neck Surgery and Audiology, Copenhagen University Hospital, Rigshospitalet, Blegdamsvej 9, Copenhagen, Denmark. E-mail: elisabeth.arndal@regionh.dk

Received: 18 June 2019 | Accepted after revision: 04 Sept 2019

Author contributions: E. Arndal, H.K. Johansen, M. Alanin, K. Aanæs, N. Høiby, K.G. Nielsen, V. Backer and C. von Buchwald developed the hypothesis and design. E. Arndal, J.A.J. Haagensen and J.A. Bartell performed the phenotypic analyses. R.L. Marvig performed the genotypic analyses. M. Alanin, K. Aanæs and C. von Buchwald performed the endoscopic sinus surgery and collected sinus samples. K.G. Nielsen collected lung samples. H.K. Johansen stored sinus and lung samples. K.G. Nielsen performed updated diagnostic review of all the patients included. E. Arndal wrote the article, supervised by C. von Buchwald, and all the authors discussed the results and critically reviewed the manuscript.

Conflict of interest: E. Arndal reports grants from Candys Foundation and Copenhagen University Hospital/ Rigshospitalet Fund, during the conduct of the study. H.K. Johansen has nothing to disclose. J.A.J. Haagensen has nothing to disclose. J.A. Bartell has nothing to disclose. R.L. Marvig has nothing to disclose. M. Alanin has nothing to disclose. K. Aanaes has nothing to disclose. N. Høiby has nothing to disclose. K.G. Nielsen has nothing to disclose. V. Backer has nothing to disclose. C. von Buchwald has nothing to disclose.

Support statement: This work was supported by Lundbeckfonden (grant: R144-A5287 to H.K. Johansen), RegionH Rammebevilling (grant: R144-A5287 to H.K. Johansen), Rigshospitalet's Research Fund (grant: 1 year introductory stipend to E. Arndal), Candys Foundation (grant: PhD stipend to E. Arndal), Rigshospitalets Rammebevilling 2015-17 (grant: R88-A3537 to H.K. Johansen), Novo Nordisk Fonden (grant: NNF12OC1015920 and NNF15OC0017444 to H.K. Johansen), Danmarks Grundforskningsfond (grant: 126 to Rasmus L. Marvig). Funding information for this article has been deposited with the Crossref Funder Registry.

\section{References}

1 Alanin MC, Nielsen KG, von Buchwald C, et al. A longitudinal study of lung bacterial pathogens in patients with primary ciliary dyskinesia. Clin Microbiol Infect 2015; 21: 1093.e1-1093.e7.

2 Moradali MF, Ghods S, Rehm BHA. Pseudomonas aeruginosa lifestyle: a paradigm for adaptation, survival and persistence. Front Cell Infect Microbiol 2017; 7: 39.

3 Hansen SK, Rau MH, Johansen HK, et al. Evolution and diversification of Pseudomonas aeruginosa in the paranasal sinuses of cystic fibrosis children have implications for chronic lung infection. ISME J 2012; 6: 31-45.

4 Aanæs K. Bacterial sinusitis can be a focus for initial lung colonisation and chronic lung infection in patients with cystic fibrosis. J Cyst Fibros 2013; 12: S1-S20.

5 Alanin MC, Johansen HK, Aanæs K, et al. Simultaneous sinus and lung infections in patients with primary ciliary dyskinesia. Acta Otolaryngol 2015; 135: 58-63.

6 Lucas JS, Barbato A, Collins SA, et al. European Respiratory Society guidelines for the diagnosis of primary ciliary dyskinesia. Eur Respir J 2017; 49: 1601090.

7 Sommer LM, Alanin MC, Marvig RL, et al. Bacterial evolution in PCD and CF patients follows the same mutational steps. Sci Rep 2016; 6: 28732-28739.

8 Ciofu O, Riis B, Pressler T, et al. Occurrence of hypermutable Pseudomonas aeruginosa in cystic fibrosis patients is associated with the oxidative stress caused by chronic lung inflammation. Antimicrob Agents Chemother 2005; 49: 2276-2282.

9 Storm DR, Rosenthal KS, Swanson PE. Polymyxin and related peptide antibiotics. Annu Rev Biochem 1977; 46: 723-763.

10 Markussen T, Marvig RL, Gómez-Lozano M, et al. Environmental heterogeneity drives within-host diversification and evolution of Pseudomonas aeruginosa. MBio 2014; 5: e01592-14.

11 Marvig RL, Sommer LM, Molin S, et al. Convergent evolution and adaptation of Pseudomonas aeruginosa within patients with cystic fibrosis. Nat Genet 2015; 47: 57-64.

12 Høiby N, Bjarnsholt T, Givskov M, et al. Antibiotic resistance of bacterial biofilms. Int J Antimicrob Agents 2010; 35: 322-332.

13 Doht F, Hentschel J, Fischer N, et al. Reduced effect of intravenous antibiotic treatment on sinonasal markers in pulmonary inflammation. Rhinology 2015; 53: 249-259.

14 Karma P, Pukander J, Penttilä M. Azithromycin concentrations in sinus fluid and mucosa after oral administration. Eur J Clin Microbiol Infect Dis 1991; 10: 856-859.

15 Alanin MC, Aanaes K, Høiby N, et al. Sinus surgery can improve quality of life, lung infections, and lung function in patients with primary ciliary dyskinesia. Int Forum Allergy Rhinol 2017; 7: 240-247.

16 Rademacher J, Ringshausen FC, Suhling H, et al. Lung transplantation for non-cystic fibrosis bronchiectasis. Respir Med 2016; 115: 60-65. 